\title{
The Establishment and Application of an Evaluation Model for Land-Use Change and Eco-Security
}

\author{
Han-Shen Chen ${ }^{1,2 *}$ \\ 1 School of Health Diet and Industry Management, Chung Shan Medical University, No.110, Sec.1, Jianguo \\ N. Rd.Taichung City 40201, Taiwan; E-Mail: allen9750@yahoo.com.tw \\ 2 Department of Medical Management, Chung Shan Medical University Hospital, No.110, Sec.1, Jianguo N. \\ Rd.Taichung City 40201, Taiwan ; E-Mails: allen9750@yahoo.com.tw \\ * Correspondence: allen9750@yahoo.com.tw; Tel.: +886-4-2473-0022
}

\begin{abstract}
Land use and cover change (LUCC) is an important method to investigate the causes of global environment change. We utilized the emergy ecological footprint (EEF) model to construct a land-use change model to be used as a systematic measuring tool for monitoring sustainable development trends. In particular, we estimated the eco-security of the Cing-jing region as a case study so that responsible agencies can use it to maintain a balance between ecological preservation and tourism development. The results indicated the following: First, the ecological environment of the Cing-jing region satisfied the safety standard in 2008-2014; however, the related indices increased considerably. Second, the grey model predicted a decrease in 2015-2024 ecological carrying capacities of Cing-jing and a large increase in capita EFs, resulting in a larger ecological deficit and higher EFI. The eco-security from 2015-2024 was higher compared to 7 years ago and is predicted to reach the Grade 2 intermediate level in 2022; thus the Cing-jing region is gradually becoming ecologically unsustainable. Strengths of our study included the use of EEF theory in a quantitative analysis of slope lands for the effective evaluation of ecological security. Finally, we expanded our research to include ecological security issues.
\end{abstract}

Keywords: Eco-security ; Land use and cover change (LUCC); Sustainability development and assessment

PACS: J0101

\section{Introduction}

In recent years, global change in land use and land cover has become a research focus [1]. The International Geosphere-Biosphere Programme, IGBP (International Human Dimensions Programme on Global Environmental Change, IHDP) jointly promoted land use and cover change (LUCC) and continued to promote the Global Land Project (GLP). The Scientific Committee on Problems of the Environment (SCOPE) was also aware of the impact of human activities on the natural environment and caused by urban expansion. We embarked on the Urban Peri-Urban Environmental Change, PU-ECH Research Project. Research on land use and cover change (LUCC) research from global to regional scale studies in different regions and temporal and spatial scales have been particularly concentrated in administrative areas, vulnerable areas and other ecologically sensitive areas [2]-[3]. Current research in this area can be divided according to regional scale into 
two types, administrative regions, such as continent, country, province, city and county [2],[4] and natural regions, such as environmentally sensitive areas and coastal areas [3],[5].

In addition, past research has focused on exploring a single most impact level, with a relative lack of analysis and evaluation of the impact of land-use development and changes on the natural environment. Therefore, the current analysis and prediction model of land use change was developed, through model simulations, as well as decomposition, analysis and synthesis of the complex socio-economic variables and their interaction with land use on natural ecosystems in order to determine trends in land-use change and spatial pattern [6]-[9]. The econometric model [10]-[11], the statistical model [12]-[17], the cellular automata model [18] as well as the integrated model [19] are the most commonly used. In recent years, interdisciplinary methods using geographic information system (GIS) to analyze the comprehensive study of spatial structure of land use changes have been developed [20]-[22].

Discussion of content including land use change process, Change mode, the driving force of the mechanism as well as environmental effects. Regional land use change model can be used to learn the correlation between regional land development, natural resources and human activities, and can investigate conditions in different situations, future land use change trends, and can help to reach a reasonable decision and to compare different decisions among programs. Studies include not only the factors at a regional scale, including land use and cover change (LUCC) effects on soil and natural disasters, as well as the impact of resource use, but also evaluate ecosystem services and use ecological evaluation methods to evaluate ecological land use and cover change (LUCC) impacts on ecosystems [23]-[25].

Areas designed as hillsides have functions in food supply, climate regulation, water resources maintenance, as well as supporting biological diversity, and are one of the important places to provide human leisure recreation. Whether from the point of environmental conservation, or from a reserved open space and sustainable management, it is worth exploring the causes and nature of the problem, as well as a viable management strategy. Past studies mostly concentrated in the disaster prevention [26]-[27] and the development of management-oriented [28]-[29]. In addition, environmental carrying capacity research has focused on evaluation methods and its applications and in particular to use some quantitative analytical methods to directly or indirectly measure the environmental carrying capacity. But studies have not explored the dynamic development trends. Thus, the objective of this study was to explore the process of land use change the impact of various factors.

Sharply in the global climate variability, desertification, biodiversity and ecosystem reduce vulnerability and other issues, in the 1972 United Nations Declaration on the Human Environment Eco-security issues included in the care objectives enshrined in the food, ecosystems and the principles of sustainable human development projects, providing a review of environmental resources and human survival and sustainable development issues in a new perspective.

The concept of eco-security was developed by the International Institute for Applied Systems Analysis (IIASA) in 1989, and included human life, health, happiness, fundamental rights, livelihood security sources, necessary resources, social order and human capacity to adapt to environmental changes and other threats. With the development of the theory of eco-security and as ecological problems have become increasingly prominent, foreign research institutions and 
scholars from different perspectives have been using different indicators and measurement system models or methods for the evaluation of the eco-security at different regional scales [30] .

However, in analyzing the characteristics of the current measurement system, or model methods, we found some deficiencies. First, to reflect the connotation of eco-security, or some measure of evaluation index model is too complex and which can reflect the dynamic changes too few indicators of sustainable development. Next, some indicators to assess or measure the model are not based on a complete evaluation index system in the construction of the model and thus it is difficult to quantify and has poor operability. Finally, some evaluation index measurement model involved the acquisition or issue of information that is difficult to put to practical use.

Current research in Ecological Safety Evaluation using quantitative evaluation methods and criteria are still at the exploratory stage. As the development of the theory of eco-security and ecological problems has become increasingly prominent, foreign research institutions and scholars from different perspectives using different indicators and measurement system models or methods for determining the eco-security of different regional scales have been evaluated through eco-security analysis, forecasting and early warning of eco-security an important reference [30] .

Studies to determine the best approach towards eco-security evaluation using quantitative evaluation methods and criteria are still at the exploratory stage, due to changes in ecosystem services due to land use change. Therefore, the use of ecosystem services from land use change estimates one economic value of the system to provide services for the human, which can be increased or decreased due to changes in the eco-security situation.

In summary, regional land use change is a central issue in recent years. This study first use the emergy ecological footprint model, with select change model of land-use parameters. Through Cing-jing regional ecosystems of measurement, load capacity and understanding of the environment of current economic activity for the environmental pressure exerted situation. Next, we built an eco-security evaluation indicator system to measure the eco-security of the Cing-jing region. Finally, we applied the grey model to predict the development trend of eco-security of the Cing-jing region as a future benefit for spatial planning, land management, and a resource conservation policy development communication tool.

\section{Materials and Methods}

\subsection{Study area}

The Cing-jing region, the research site used in this study, is located in central Taiwan. The Cing-jing region is a popular location for leisure and recreation attractions, and is an important asset to develop international tourism and attract foreign tourists, by virtue of its distinctive terrain and landscape combined with the unique cultural heritage of the indigenous Truku people. However, with restricted to land usage division, Cing-jing has no land for building hotels except for those built in early stages of development, such as Cing-jing Guest Hotel, as well as cabins and camping sites that have already been planned. In order to meet tourist demand, local residents provide accommodations to tourists in the way of bed and breakfasts (B\&B). However, mountain areas have deep terrain and fragile geology, together with heavy and concentrated rainfall. Building new facilities will definitely have severe impacts on the ecological environment as well as the 
maintenance of water and land resources. Therefore, sustainable development of tourist resources and lower recreational impact are urgently needed so that the hotel industry can make use of resources effectively, to reduce cost and increase operational efficiency, to ensure tourist industry can develop under the principle of sustainable development and to benefit the conservation of ecological systems.

Our evaluation model can be used in other mountain ecosystems, as the the topography, ecological environment, and existing facilities of Cing-jing have similar attributes as other mountainous areas in Taiwan.

\subsection{Method}

\subsubsection{Land use change patterns}

In this study, we applied the emergy ecological footprint method to measure the area of land use change in the Cing-jing region. The emergy ecological footprint applies the emergy theory, which includes actual consumption of six types of biologically productive land (fossil energy land, arable land, grassland, forest, building land and waters) and the amount of resources provided. It can be added into common metrics - energy value, and can incorporate energy value density (based on the value of the number of all renewable energy resources per unit area) and transform an easy area concept that people can understand.

The emergy ecological footprint is divided into two biological resources and energy. Biological resources include agricultural products, forest products, livestock products, aquatic products, and respective four types of biological productivity of land corresponding to the type, namely farmland, woodland, grassland waters. Energy included coal, crude oil, natural gas, electric power, and building land, corresponding to the remaining land and fossil energy. The biological productivity of land consumption types, the physical quantity corresponding to the number of energy values and the value of the number of $\left(c_{i}\right)$ available per capita consumption, divided by $\left(P_{1}\right)$, can be obtained to find per capita Ecological Footprint (ef). The formula as defined in formula (2-1):

$$
\text { ef }=\sum_{i=1}^{n} a_{i}=\sum_{i=1}^{n} c_{i} / P_{1}
$$

In the formula, $i$ is the type of resource consumption; ef is per capita emergy ecological footprint; $a_{i}$ is per capita consumption of resources corresponding to the $i$-th area; $c_{i}$ is the $i$-th value per capita energy consumption of the resource, unit is sej; $P_{1}$ is regional energy density value , $P_{1}=$ the area to obtain the total energy value / regional area, in units of sej / $\mathrm{m}^{2}$.

Emergy ecological footprint of ecological carrying capacity is all renewable energy resources in the study region and the global average value emergy density ratio. In calculating the energy value of the regional ecological carrying capacity, first, the amount that a region's population can obtain renewable resources, including solar, wind, rain and potential chemical energy, and energy value of rotation of the Earth should be calculated. In addition, in order to avoid double counting, the 
maximum value is taken as the regional one, and the number available resources per capita energy supply (e), divided by the energy density value $\left(P_{2}\right)$, per capita energy value can be obtained ecological carrying capacity (ec). The formula as defined in formula (2-2):

$$
e c=e / P_{2}
$$

In the formula, $e c$ is the per capita emergy ecological carrying capacity $; e$ is the supply of energy resources per capita amount of value; $P_{2}$ is global energy density, units with $P_{1}$, calculated as follows: $P_{2}=$ The total energy value of the Earth / Earth area = Earth's biosphere major energy values of the reference value is $1.583 \times 1025 \mathrm{sej} / \mathrm{a}$.

When EFI is equal to 1, ecological carrying capacity supply and demand are balanced and the ecosystem is in a critical state of sustainable development. Since emergy ecological footprint calculations are based on official statistics of regional, national and international government departments, more objective accuracy is also important in determining environmental carrying capacity. This research constructed indicators to assess the environmental carrying capacity as follows:

\subsection{2 the ecological pressure index}

In this study, we used the Ecological Footprint Method to measure the ecological resources of the Cing-jing region to determine if it exceeded the carrying capacity of the land in the area. EFI is defined as the renewable resources per capita Ecological Footprint (ef') and emergy ecological carrying capacity ratio $(e c)$, this index carried the degree of the Cing-jing regional ecological environment. The formula as defined in formula (2-3):

$$
\mathrm{EFI}=e f^{\prime} / e c
$$

In the formula , EFI is the ecological pressure index; ef' is people can update resource emergy ecological footprint; $e c$ is per capita emergy ecological carrying capacity.

When EFI is equal to 1, energy ecological carrying capacity supply and demand are balanced and ecosystems are in a critical state of sustainable development. When EFI is less than 1, emergy ecological carrying capacity is greater than the ecological footprint and the ecosystem load will be normal and in the sustainable development of the state. When EFI is greater than 1, the Ecological Footprint is greater than the emergy ecological carrying capacity, and thus human consumption has exceeded the ecological carrying capacity, in departure of a sustainable development state.

2.2.3 Corresponding quotiety of ecological

Corresponding quotiety of ecological (DS) was used to measure the functional relationship between Cing-jing regional development and the ecological environment, in order to compensate for the lack of ecological deficit. The formula is defined in formula (2-4): 


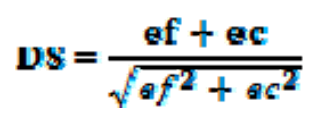

In the formula , DS is the regional per capita corresponding quotiety of ecological; ef is the per capita emergy ecological footprint; $e c$ is the per capita ecological carrying capacity.

DS as an important indicator of ecological harmony and the react ecological sustainability status in Cing-jing area, it can be obtained by the formula: When the DS closer to 1, Cing-jing area emergy ecological footprint is higher than the ecological carrying capacity, when the ecological harmony worse, the lower the degree of regional development; Conversely, Cing-jing area emergy ecological footprint and ecological capacity balance, coordination in the best state. Thus, the Corresponding quotiety of ecological in response to a certain extent the eco-security of the region.

Per capita EECC and per capita EEF to were used to calculate the 2008-2014 EFIs of the research area (Table 1). We found that EFI increased from 1.08 in 2008 to 2.14 in 2014; thus the ecosystem is continually stressed and eco-security is threatened.

Table 1. The 2008-2014 EFI, DS and ES of Cimg-jing region

\begin{tabular}{cccccccc}
\hline Year & 2008 & 2009 & 2010 & 2011 & 2012 & 2013 & 2014 \\
\hline EFI & 1.08 & 1.34 & 1.36 & 1.43 & 1.80 & 1.97 & 2.14 \\
DS & 1.92 & 1.76 & 1.68 & 1.52 & 1.47 & 1.25 & 1.13 \\
ES level & 2 & 2 & 2 & 2 & 2 & 2 & 2 \\
ES evaluation & Safe & Safe & Safe & Safe & Safe & Safe & Safe \\
\hline \hline
\end{tabular}

Source: Data collected and organized by this study.

\section{Results}

Based on the fundamental data of the grey prediction model, we predicted the 2015-2024 per capita EEFs and per capita EECCs of the Cing-jing region (Table 2). Although the 2015-2024 per capita EECCs of the Cing-jing region increased slightly, the 2020-2024 capacities decreased. In addition, the 2015-2024 per capita EEFs grew substantially. This is expected to enlarge the regional per capita ecological deficit and render the development of the entire region unsustainable, thereby further endangering the ecological environment of the region.

Based on the prediction results for 2015-2024, the 2015-2024 per capita ecological deficit and surplus values can be attained (Table 2). The results showed that the 2015-2024 ecological deficits of the Cing-jing region gradually increased. In future development of the Cing-jing region, because EF and per capita demand continually increase annually while natural supplies continue to decrease, the per capita ecological deficit in 2024 is anticipated to be $-23.03 \mathrm{hm} 2 / \mathrm{cap}$. This means that human demand will exceed the load capacity of natural systems considerably, thereby causing the ecological environment to deteriorate. In addition, in 2015-2024, although the eco-security level of the Cing-jing cingregion satisfied the safety standard, the EFI increased, which will induce a rapid 
deterioration of the ecological environment.

By using EFI-related data and integrating these data with eco-security standards, eco-security of 2015-2024 can be obtained (Table 2). In 2018, the Cing-jing region is expected to reach the Grade 2 intermediate level, and the early-warning level will increase rapidly over time. The reason may be that tourist demand for resources and services (e.g., bus service, road constructions, and waste production) increase as the number of people increase. Therefore, we suggest that the managers of the Cing-jing region continue to track the recreational demands and usage conditions of recreational facilities and resources of tourists by using satisfaction surveys. Thus a basis for revising EEF calculation methods and for precisely evaluating the capacity for tourist load in the Cing-jing region can be attained.

Table 2. The evaluation and early warning stage of ES

\begin{tabular}{|c|c|c|c|c|c|c|c|c|}
\hline \multirow{2}{*}{ Year } & \multirow{2}{*}{$\begin{array}{c}\text { Per } \\
\text { capita } \\
\text { EECC }\end{array}$} & \multirow{2}{*}{$\begin{array}{c}\text { Per } \\
\text { capita } \\
\text { EEF }\end{array}$} & \multirow{2}{*}{$\begin{array}{c}\text { Ecological } \\
\text { deficit/ } \\
\text { surplus }\end{array}$} & \multicolumn{3}{|c|}{ EFI } & \multicolumn{2}{|c|}{$\begin{array}{c}\text { ES early } \\
\text { warning state }\end{array}$} \\
\hline & & & & Index & Grade & $\begin{array}{l}\text { Safety } \\
\text { Status }\end{array}$ & Level & Status \\
\hline 2015 & 3.92 & 9.24 & -5.32 & 2.36 & 2 & Safe & 1 & Mild \\
\hline 2016 & 3.95 & 10.40 & -6.45 & 2.63 & 2 & Safe & 1 & Mild \\
\hline 2017 & 3.99 & 11.71 & -7.72 & 2.93 & 2 & Safe & 1 & Mild \\
\hline 2018 & 4.03 & 13.18 & -9.15 & 3.27 & 2 & Safe & 1 & Mild \\
\hline 2019 & 4.07 & 14.83 & -10.76 & 3.64 & 2 & Safe & 1 & Mild \\
\hline 2020 & 4.02 & 16.70 & -12.68 & 4.15 & 2 & Safe & 1 & Mild \\
\hline 2021 & 3.96 & 18.79 & -14.83 & 4.74 & 2 & Safe & 1 & Mild \\
\hline 2022 & 3.93 & 21.15 & -17.22 & 5.38 & 2 & Safe & 2 & $\begin{array}{l}\text { Inter- } \\
\text { mediary }\end{array}$ \\
\hline 2023 & 3.87 & 23.80 & -19.93 & 6.15 & 2 & Safe & 2 & $\begin{array}{l}\text { Inter- } \\
\text { mediary }\end{array}$ \\
\hline 2024 & 3.76 & 26.79 & -23.03 & 7.13 & 2 & Safe & 2 & $\begin{array}{c}\text { Inter- } \\
\text { mediary }\end{array}$ \\
\hline
\end{tabular}

Source: Data collected and organized by this study.

\section{Discussion}

This study applied the EEF method and adopted a comprehensive evaluation method, involving the EFI and the grey prediction model, to conduct evaluation, prediction, and early warning studies of eco-security for the Cing-jing region. We obtained the following conclusions.

We employed the EEF model to calculate ecological deficit and surplus. Moreover, EFIs were obtained by comprehensively analyzing and evaluating the 2008-2014 eco-security. The results indicated that during 2008-2014, the ecological environment of the Cing-jing region was in a safe state; however, the related indices increased annually.

The grey model was adopted to predict the 2015-2024 EEFs and ecological carrying capacities of the Cing-jing region. Next, ecological deficit values and EFIs were calculated to predict the eco-security trend and relevant early warning status. The results showed that the 2015-2024 ecological carrying capacities of the Cing-jing region decreased, the regional per capita EF increased 
considerably, and the ecological deficit and EFI increased. The results of eco-security evaluation revealed that between 2015 and 2024, the eco-security level of the Cing-jing region met the safety condition. However, combined with the EFIs, the eco-security status exhibited a declining trend, which is expected to cause the ecological environment to deteriorate. The eco-security level during 2015-2024 was greater than that of 7 years ago and is anticipated to reach the Grade 2 intermediate level in 2022. Moreover, the early warning level will also increase rapidly over the years.

Based on the conclusions above, this paper suggests, in order to effectively control biocapacity and to achieve the goal of conservation and sustainable development of the Cing-jing region, the regulations on land planning and use should continue being used and a more explicit conservation program geared towards Cing-jing's regional resources characteristics should be formulated in order to attain long-term preservation of the unique and scenic natural environments, biodiversity and historical relics. In addition, the management office should designate special persons for patrol and inspection to keep a strict control of recreational activities in the park and prevent improper behavior of tourists, which may damage or contaminate the environment, and should at the same time demolish illegal buildings or facilities. As well as meeting the demand for transport and recreation by appropriately widening and renovating the road so as to ensure smooth transport, it is also suggested that $\mathrm{CO}_{2}$-emission-reduction and energy-saving leisure tourism be popularized. People should be encouraged to use mass transportation means or ride low-carbon, low-power-consumption and low-pollution bicycles, while prudently assessing the safety of the geographic environment and road.

\section{Conclusions}

The environmental impact of tourists can vary in space and in time, as tourist activity is influenced by such factors as climate, holidays and celebration events. The frequency of tourist activities and the concentration of tourists can change the ecosystem (e.g., concentrated excessive emission of pollutants can have impacts) as well as cause permanent damage. However EEF is calculated on an annual basis and these variations in impacts are thus not considered.

Acknowledgments: I am thankful to the Ministry of Science and Technology (Republic of China, Taiwan) for financially supporting this research project (grant number MOST 104-2410-H-040-010)

Conflicts of Interest: The authors declare no conflict of interest. 


\section{References}

1. Xiao, J., Shen, Y., Ge, J., Tateishi, R., Tang, C., Liang, Y., \& Huang, Z.(2006).Evaluating urban expansion and land use change in Shijiazhuang, China, by using GIS and remote sensing. Landscape and Urban Planning 75: 69-80.

2. Cameron, G., Muellbauer, J. and Snieker, J.(2002). A study in structural change: relative earnings in Wales since the 1970s. Regional Studies, 36(1):1-11.

3. Dubroeucq, D. and Livenais, P.(2004), Land cover and land use changes in relation to social evolution-a case study from Northern Chile. Journal of Arid Environments, 56(2):193-211.

4. StePhenne, N.and Lambin, E. E.(2001). A dynamic simulation model of land-use changes in Sudanosahelian countries of Africa (SALU). Agriculture, Ecosystems and Environment, 85:145-161.

5. Kok, K., Veldkamp, A.(2001), Evaluating impact of spatial scales on land use pattern analysis in Central America. Agriculture, Ecosystems and Environment, 85:205-221.

6. Petus, C., Lewis, M. and White, D. (2013). Monitoring temporal dynamics of Great Artesian Basin wetland vegetation, Australia, using MODIS NDVI. Ecological Indicators, 34:41-52.

7. Julian, J. P., Wilgruber, N. A., Beurs, K. M. D., Mayer, P. M. and Jawarnehd, R. N., (2015). Long-term impacts of land cover changes on stream channel loss. Science of the Total Environment, 537:399-410.

8. Abrantes, P., Fontes, I., Gomes, E. and Rocha. J.(2016). Compliance of land cover changes with municipal land use planning: Evidence from the Lisbon metropolitan region (1990-2007). Land Use Policy, 51:120-134.

9. Chen, N., Ma, T. and Zhang, X. (2016). Responses of soil erosion processes to land cover changes in the Loess Plateau of China: A case study on the Beiluo River basin. Catena,136:118-127.

10. Liu, Y., Huang, X., Yang, H. and Zhong, T. (2014). Environmental effects of land-use / cover change caused by urbanization and policies in Southwest China Karst area - A case study of Guiyang. Habitat International, 44:339-348.

11. Su, S., Zhou, X., Wan, C., Li, Y. and Kong, W. (2016). Land use changes to cash crop plantations: crop types, multilevel determinants and policy implications. Land Use Policy, 50:379-389.

12. Tomaz, C., Alegria, C., Monteiro, J. M. and Teixira. M. C. (2013). Land cover change and afforestation of marginal and abandoned agricultural land: A 10 year analysis in a Mediterranean region. Forest Ecology and Management, 308:40-49.

13. Trincsi, K., Pham, T. H. and Turner S. (2014). Mapping mountain diversity: Ethnic minorities and land use land cover change in Vietnam's borderlands. Land Use Policy, 41:484-497

14. Du, S., Wang, Q. and Guo, L. (2014). Spatially varying relationships between land-cover change and driving factors at multiple sampling scales. Journal of Environmental Management, 137:101-110.

15. Xiong, X., Grunwald. S., Myers. D. B., Ross. C. W., Harris. W. G.and Comerford. N. B. (2014). Interaction effects of climate and land use / land cover change on soil organic carbon sequestration. Science of the Total Environment, 493:974-982.

16. Deng, Y. and Srinivasanb, S. (2016). Urban land use change and regional access: A case study in Beijing, China. Habitat International,51:103-113.

17. Beyene, F.(2016). Land use change and determinants of land management: Experience of pastoral and agro-pastoral herders in eastern Ethiopia. Journal of Arid Environments,125:56-63. 
18. Halmy, M. W. A., Gessler, P. E., Hicke, J. A.and Salem, B. B.(2015). Land use / land cover change detection and prediction in the north-western coastal desert of Egypt using Markov-CA. Applied Geography, 63:101-112.

19. Promper, C., Puissant, A., Malet, J. and Glade, T. (2014). Analysis of land cover changes in the past and the future as contribution to landslide risk scenarios. Applied Geography, 53:11-19.

20. Grecchi, R. C., Hugh, Q., Gwyn, J., Benie, G. B., Formaggio, A. R. and Fahl, F. C. (2014). Land use and land cover changes in the Brazilian Cerrado: A multidisciplinary approach to assess the impacts of agricultural expansion. Applied Geography, 55: 300-312.

21. Wan, L., Zhang. Y., Zhang, X., Qi, S. and Na, X., (2015). Comparison of land use / land cover change and landscape patterns in Honghe National Nature Reserve and the surrounding Jiansanjiang Region, China. Ecological Indicators,51:205-214.

22. Shooshtari, S. J. and Gholamalifard, M. (2015). Scenario-based land cover change modeling and its implications for landscape pattern analysis in the Neka Watershed, Iran. Remote Sensing Applications. Society and Environment,1:1-19.

23. Burkhard, B., Kroll, F., Müller F. and Windhorst, W. (2009). Landscapes, capacities to provide ecosystem services: A concept for land-cover based assessments. Landscape Online, 15:1-22.

24. Vihervaara, P., Kumpula,T., Tanskanen, A., and Burkhard, B.(2010).Ecosystem services-a tool for sustainable management of human-environment systems. Case study Finnish Forest Lapland. Ecological Complexity, 7(3):410-420.

25. Frank, S., Fürst, C., Koschke, L. and Makeschin, F. (2011). A contribution towards a transfer of the ecosystem services concept to landscape planning using landscape metrics. Ecological Indicators, 21: 30-38.

26. Chen, S., Ferng, J., Wang, Y., Wu, T. and Wang, J. (2008). Assessment of disaster resilience capacity of hillslope communities with high risk for geological hazards. Engineering Geology, 98(3-4): 86-101.

27. Ho, K. K. S. and Ko, F. W. Y. (2009). Application of quantified risk analysis in landslide risk management practice: Hong Kong experience. Georisk: Assessment and Management of Risk for Engineered Systems and Geohazards, 3(3):134-146.

28. Baban, S. M. J., Thomas, D., Canisius, F and Sant, K. J.(2008). Managing development in the hillsides of Trinidad and Tobago using geoinformatics. Sustainable Development, 16(5): 314-328.

29. Juan, Y.-K., Castro, D. and Roper, K.(2010). Decision support approach based on multiple objectives and resources for assessing the relocation plan of dangerous hillside aggregations. European Journal of Operational Research, 202(1): 265-272.

30. Chen, H.S., Chen, C.Y., Chang, C.T. and Hsieh, T.(2014). The Construction and Application of a Carrying Capacity Evaluation Model in a National Park. Stochastic Environmental Research and Risk Assessment, 28(6):1333-1341.

(C) 2016 by the authors; licensee Preprints, Basel, Switzerland. This article is an open access article distributed under the terms and conditions of the Creative Commons by Attribution (CC-BY) license (http://creativecommons.org/licenses/by/4.0/). 